\title{
The molecular mechanism of the anticancer effect of atorvastatin: DNA microarray and bioinformatic analyses
}

\author{
YAN GAO ${ }^{1,3^{*}}$, XUE-CHUN LU ${ }^{2 *}$, HONG-YING YANG ${ }^{4}$, XIAN-FENG LIU $^{1}$, JIAN CAO $^{1}$ and LI FAN ${ }^{1}$ \\ ${ }^{1}$ First Geriatric Cardiology Division and ${ }^{2}$ Department of Geriatric Hematology, Chinese PLA General Hospital, \\ Beijing 100853; ${ }^{3}$ Third Department of Cadre Ward, General Hospital of Jinan Military Region, Jinan 250031; \\ ${ }^{4}$ Department of Cardiology, The Fourth People's Hospital of Jinan, Jinan 250032, P.R. China
}

Received April 5, 2012; Accepted June 14, 2012

DOI: $10.3892 /$ ijmm.2012.1054

\begin{abstract}
The aim of this study was to identify the molecular mechanisms and biological pathways associated with the anticancer effects of atorvastatin. For this purpose, we conducted cell-based microarray and bioinformatic analyses to determine the effect of atorvastatin exposure on endothelial cell response. The results of bioinformatic analysis performed using the Connectivity Map (cMap) to examine the atorvastatin-induced changes in gene expression in the human umbilical vein endothelial cell line, EA.hy926, indicated that treatment with $10 \mu \mathrm{M}$ of atorvastatin for $24 \mathrm{~h}$ upregulated the expression of 295 genes and downregulated the expression of 354 genes by 2 -fold compared to the control treatment. The gene set enrichment analysis (GSEA), the Database for Annotation, Visualization and Integrated Discovery (DAVID) pathway analysis, and Gene Ontology (GO) analysis of differentially expressed genes revealed that Kruppel-like factors (KLFs) and cell cycle-related genes were the genes most significantly affected by atorvastatin treatment. The upregulation of KLFs and the downregulation of the cell cycle-related genes, including cyclin (CCN)A2, CCNE2, CCNB1 and CCNB2, were validated by real-time polymerase chain reaction (RT-PCR). A comparison of the gene expression profile of atorvastatin-treated cells with that of the control cells and with that of 6,100 compounds in the cMap database revealed that the profile of atorvastatin-treated cells was highly similar to that of histone deacetylase (HDAC) inhibitor-treated cells. Therefore, these results suggest that atorvastatin acts as an HDAC, a G1/S (start) and a G2/M (mitosis) cell cycle inhibitor. These findings provide evidence of the feasibility of the use of atorvastatin as an anticancer drug.
\end{abstract}

Correspondence to: Dr Li Fan, First Geriatric Cardiology Division, Chinese PLA General Hospital, 28 Fuxing Road, Beijing 100853, P.R. China

E-mail: fanli301@163.com

*Contributed equally

Key words: atorvastatin, bioinformatics, connectivity map, Database for Annotation, Visualization and Integrated Discovery, DNA microarray, Gene Ontology analysis, gene set enrichment analysis, Kruppel-like factor

\section{Introduction}

As a cholesterol-lowering therapy, the administration of the 3-hydroxy-3-methylglutaryl co-enzyme (HMG-CoA) reductase inhibitors, commonly known as statins, has become increasingly widespread. Statins have been found to exert biological effects at the cellular and molecular levels that contribute not only to their cholesterol-lowering effect but also to their cholesterol-independent pleiotropic mechanisms (1). These properties, together with the high safety profile of statins, have fueled research into the potential of expanding indications for statin therapy for certain populations, such as lower-risk individuals without hyperlipidemia but with elevated high-sensitivity C-reactive protein levels. Among these studies, the Justification for the Use of Statins in Primary Prevention (JUPITER) trial found that the administration of rosuvastatin as a form of primary prevention significantly reduced the incidence of major cardiovascular disease $(2,3)$.

Increased research into statin therapy has initiated a controversy that has endured for over a decade, mainly of whether an association exists between statin use and cancer. The findings of studies that have investigated this correlation have been conflicting. Among them, the Simvastatin and Ezetimibe in Aortic Stenosis (SEAS) trial reported a higher incidence of cancer in an experimental group that underwent simvastatinezetimibe therapy compared to a control group (4). By contrast, a retrospective cohort analysis of approximately 46,000 propensity-matched pairs of 11 million adult Americans demonstrated no statistically significant increased risk of cancer with statin therapy (5), nor did a study of elderly patients (6). Interestingly, evidence that inspired our aim to determine the true correlation between statins and cancer was based on a study that found a correlation between lovastatin therapy and a reduction in colorectal cancer risk in postmenopausal women (7). Compared to the conflicting clinical results obtained by these previous studies, a number of recent studies on human cancer cell lines and animal tumor models have obtained more consistent results. Based on these findings, it is hypothesized that statins possess chemopreventive properties that arrest cell cycle progression, leading to the induction of apoptosis (1), inhibition of cell proliferation (8), or inhibition of angiogenesis (9).

As the growth and metastasis of most tumors are angiogenesis-dependent, tumor cell proliferation, as well as 
endothelial cell proliferation and activity, both of which are involved in microvasculature formation, control the progress of tumor development (10). In order to discover the molecular mechanism of the antitumor effect of atorvastatin, this study conducted cell-based microarray analysis using the EA.hy.926 human endothelial cell line to obtain a better understanding of the possible effects of atorvastatin exposure on endothelial cell responses and to identify the molecular mechanisms and biological pathways associated with the anticancer effects of atorvastatin. We applied the gene set enrichment analysis (GSEA) (v2.07, Broad Institute), the Database for Annotation, Visualization and Integrated Discovery (DAVID) (Bioinformatics Resources, 6.7), and the Connectivity Map (cMap) to analyze the microarray data. GSEA is a computational method used to determine whether a pre-defined set of genes shows statistically significant differences between 2 biological states (11). DAVID extracts biological features associated with large gene lists (12). The cMap is a collection of gene expression profiles from cultured human cell lines that are treated with diverse bioactive small molecules. The current collection (build 02) contains data for 6,100 treatment instances representing 1,309 discrete small molecules, which help in understanding 'connections' among drugs, genes and diseases, and find new uses for existing drugs (13).

\section{Materials and methods}

Cell culture. The human umbilical vein endothelial cell line, EA.hy926, was purchased from the Cell Bank of the Institute of Cellular Biology at the Chinese Academy of Sciences, Shanghai, China. Cells were cultured in Dulbecco's modified Eagle's medium (DMEM) supplemented with $10 \%$ fetal bovine serum (FBS; Gibco, Gaithersburg, MD, USA) and 1\% penicillin/streptomycin at $37^{\circ} \mathrm{C}$ in a humidified atmosphere containing $95 \% \mathrm{O}_{2}$ and $5 \% \mathrm{CO}_{2}$. Atorvastatin (NICPBP, Beijing, China) was dissolved in stock $10 \mathrm{mM}$ dimethyl sulfoxide (DMSO; Sigma, St. Louis, MO, USA) and added to the cells at the indicated concentrations for the entire incubation period. The final concentration of DMSO never exceeded $0.1 \%$.

Analysis of differential gene expression. After EA.hy926 cells had been collected subsequent to incubation with atorvastatin $(10 \mu \mathrm{M})$ or the control (DMSO) for $24 \mathrm{~h}$, the Affymetrix U133A Plus 2.0 GeneChip (CapitalBio Corp., Beijing, China) was used to assay each group of cells 3 times to determine the gene expression profiles. Total-RNA was extracted from $5 \times 10^{6}$ cells with TRIzol ${ }^{\circledR}$ (Invitrogen, Grand Island, NY, USA) according to the manufacturer's instructions and purified using RNeasy spin columns (Qiagen, Hilden, Germany). The quality of the RNA was assessed by performing gel electrophoresis and by determining the optical density (OD) 260/OD 280 ratio. After cDNA had been synthesized from DNase-treated totalRNA (7 $\mu \mathrm{g})$ using the Superscript II double-stranded cDNA synthesis kit (Applied Biosystems, Carlsbad, CA, USA), it was used for in vitro transcription in the presence of biotin-labeled ribonucleotides (biotin-11-CTPs und biotin-16-UTPs) to yield biotin-labeled cRNA. The biotin-labeled RNA fragments were then hybridized to the probe array during $16 \mathrm{~h}$ of incubation before staining the array with streptavidin-phycoerythrin conjugate and scanning it using the GeneChip ${ }^{\circledR}$ Scanner 3000. Finally, the hybridization results were analyzed using the Affymetrix GeneChip Operating Software Version 1.4.

GSEA. Based on the entire microarray profiles using predefined gene sets, GSEA applies novel computational methods to detect pathways that may serve as targets for novel therapeutics (11). Two categories of pre-defined gene sets in the Molecular Signatures Database (MSigDB) were selected for analysis: the $\mathrm{C} 4$ set, a computational gene set defined by mining large collections of cancer-oriented microarray data that includes cancer gene neighborhoods and cancer modules, and the C5 set, a Gene Ontology (GO) molecular function gene set derived from the Molecular Function Ontology database (14). The gene sets included in the analysis were limited to those that contained between 10 and 500 genes. Permutation was conducted 1,000 times according to default-weighted enrichment statistics and by using a signal-to-noise metric to rank genes according to their differential expression levels across the atorvastatin and DMSO groups. Significant gene sets were defined as those with a nominal P-value $<0.05$.

DAVID. The probe sets of overexpressed and underexpressed genes in the atorvastatin and DMSO groups were uploaded to DAVID maintained by the National Institute of Allergy and Infectious Diseases (NIAID) (12). Using the DAVID functional annotation tool version 6.7, gene annotation enrichment analysis, functional annotation clustering, BioCarta and KEGG pathway mapping, gene-disease correlation analyses were performed.

cMap analysis. cMap only includes gene data from Affymetrix HG-U133A probe sets. The gene sets belonging to Affymetrix HG-U133A Plus 2.0 probe sets, but not to Affymetrix HG-U133A probe sets were excluded, and the remaining gene sets were further processed.

All mapped probe sets remaining after the above exclusion process and indicating a 2-fold difference in gene upregulation or downregulation between the atorvastatin and DMSO groups were entered into the cMap (15). In the latest dataset version (build 02) of the cMap, which contains 6,100 expression profiles representing 4 cultured human cell lines (MCF7 breast cancer epithelial cell line, PC3 prostate cancer cell line, HL60 nonepithelial leukemia cell line and SKMEL5 melanoma cell line) treated with 1,309 bioactive small molecules (13), gene expression profiles of instance sets are rank ordered by descending connectivity scores as calculated by Kolmogorov-Smirnov statistics. Permutation tests were performed to estimate the significance of the instance sets ranked by the connectivity scores, with negative scores indicating that treatment with a compound altered the probe sets in a manner opposite to one if they were altered by atorvastatin treatment.

Real-time polymerase chain reaction (RT-PCR) analysis. RNA was extracted using TRIzol and quantified by measuring the absorbance at $260 \mathrm{~nm}$ for reverse transcription analysis using the One Step RT-PCR kit (Promega, Madison, WI, USA) according to the manufacturer's instructions. After cDNA samples $(2 \mu \mathrm{l})$ were amplified in $20 \mu \mathrm{l}$ of $1 \mathrm{X} \mathrm{SYBR}{ }^{\circledR}$ Green PCR Master Mix (Applied Biosystems), RT-PCR was 
Table I. Primers for real-time quantitative RT-PCR.

\begin{tabular}{|c|c|c|}
\hline Gene name & Primer sequences (F, forward; $R$, reverse) & Amplicon size (bp) \\
\hline GAPDH & $\begin{array}{l}\text { F: TGCGCAGAAAACAAGATGAG } \\
\text { R: CACCTTCACCGTTCCAGTTT }\end{array}$ & 114 \\
\hline KLF2 & $\begin{array}{l}\text { F: GCAAACGCACCGCCACTCACACCT } \\
\text { R: CTTCCAGCCGCAGCCGTCCCAGTT }\end{array}$ & 140 \\
\hline KLF3 & $\begin{array}{l}\text { F: GTGATTATGATGGATGCAACAAA } \\
\text { R: TTCATCAGACCGAGCAAACTT }\end{array}$ & 134 \\
\hline KLF4 & $\begin{array}{l}\text { F: GCGGGCTGCGGCAAAACCTACAC } \\
\text { R: CATCCACAGCCGTCCCAGTCACAG }\end{array}$ & 104 \\
\hline KLF6 & $\begin{array}{l}\text { F: AGCTCCTCTGTCACCTCCAC } \\
\text { R: CAGCTCCCCGGGCACGCAA }\end{array}$ & 87 \\
\hline KLF7 & $\begin{array}{l}\text { F: GTTTTGCACGAAGCGATGAG } \\
\text { R: ATGTGGAGGGCAAGATGGTC }\end{array}$ & 118 \\
\hline KLF9 & $\begin{array}{l}\text { F: GAAACACGCCTCCGAAAAG } \\
\text { R: TCACCTGTATGCACTCTGTAATGG }\end{array}$ & 105 \\
\hline KLF13 & $\begin{array}{l}\text { F: TCGGGAGAATACAGCTCCGATTTCT } \\
\text { R: TGTCCATAAAGGTACTGAAGCTG }\end{array}$ & 112 \\
\hline CCNB2 & $\begin{array}{l}\text { F: GCACATGGCCAAGAATGTGGTG } \\
\text { R: TCAGTGGGGAGGCAAGGTCTT }\end{array}$ & 149 \\
\hline CCNB1 & $\begin{array}{l}\text { F: ACATGGTGCACTTTCCTCCTTCTC } \\
\text { R: GTAGAGTTGGTGTCCATTCACC }\end{array}$ & 93 \\
\hline CCNA2 & $\begin{array}{l}\text { F: TTGCTGGAGCTGCCTTTCATTTAG } \\
\text { R: CCAGGGTATATCCAGTCTTTCGTA }\end{array}$ & 90 \\
\hline CCNE2 & $\begin{array}{l}\text { F: GGATGGTACCTTTTGTCAATGTAG } \\
\text { R: AATTTACTTCCTCCAGCATAGCC }\end{array}$ & 116 \\
\hline
\end{tabular}

performed on duplicate samples using the ABI PRISM ${ }^{\circledR}$ 7300 Real-Time PCR System (Applied Biosystems) with the following cycling parameters: initial denaturation at $95^{\circ} \mathrm{C}$ for $10 \mathrm{~min}, 40$ cycles of denaturation at $95^{\circ} \mathrm{C}$ for $15 \mathrm{sec}$, and annealing/extension at $61^{\circ} \mathrm{C}$ for $31 \mathrm{sec}$. Data were normalized to human glyceraldehyde-3-phosphate dehydrogenase (GAPDH) mRNA levels as an endogenous control and expressed relative to the DMSO-treated control using the $2^{-\Delta \Delta \mathrm{Ct}}$ method. The primers (Table I) were designed using Primer Express ${ }^{\circledR}$ Primer Design Software V3.0 (Applied Biosystems).

Microarray analysis. To identify genes that were differentially expressed in the atorvastatin and DMSO groups, the expression level of each gene was transformed into a $\log 2$ base. The SAM algorithm was applied to the gene expression arrays from the 3 replicates of each group to perform cluster analysis using Cluster 3.0 and TreeView software.

\section{Results}

Expression profiles of the atorvastatin and DMSO groups. The 3 replicates within the atorvastatin group were found with a high

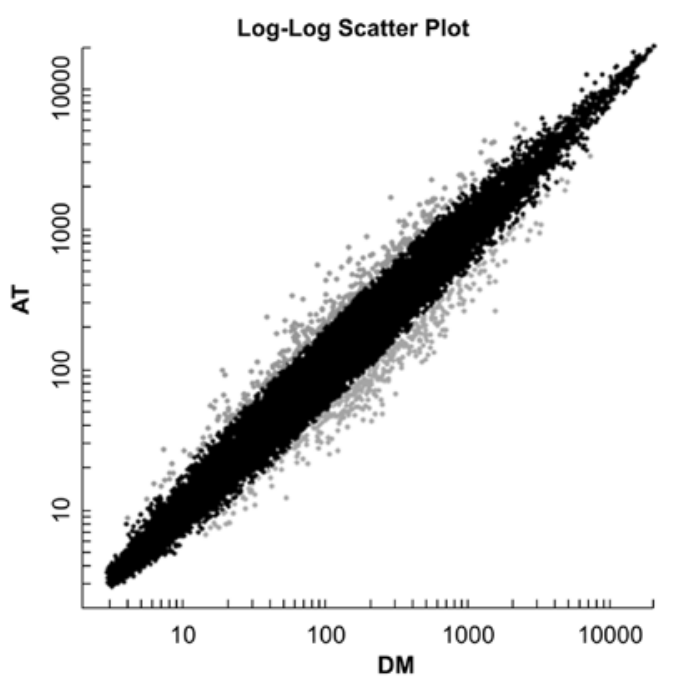

Figure 1. Log-log scatter plot of gene expression of EA.hy926 cells following atorvastatin treatment. The $\mathrm{x}$ - and the $\mathrm{y}$-axis show the microarray expression values of the dimethyl sulfoxide and atorvastatin groups, respectively. The grey dots above the cluster represent the upregulated genes and the ones below the cluster represent the downregulated genes, $>2$-fold compared to the control treatment in the 3 arrays (18,985 gene features). 

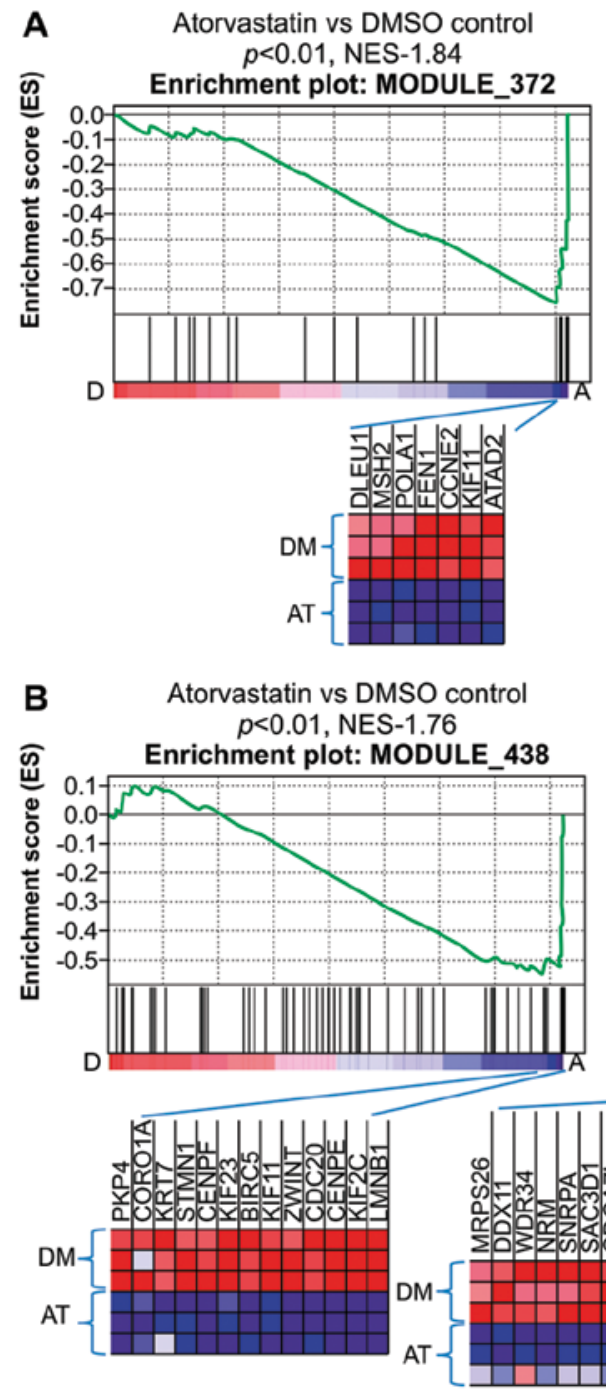
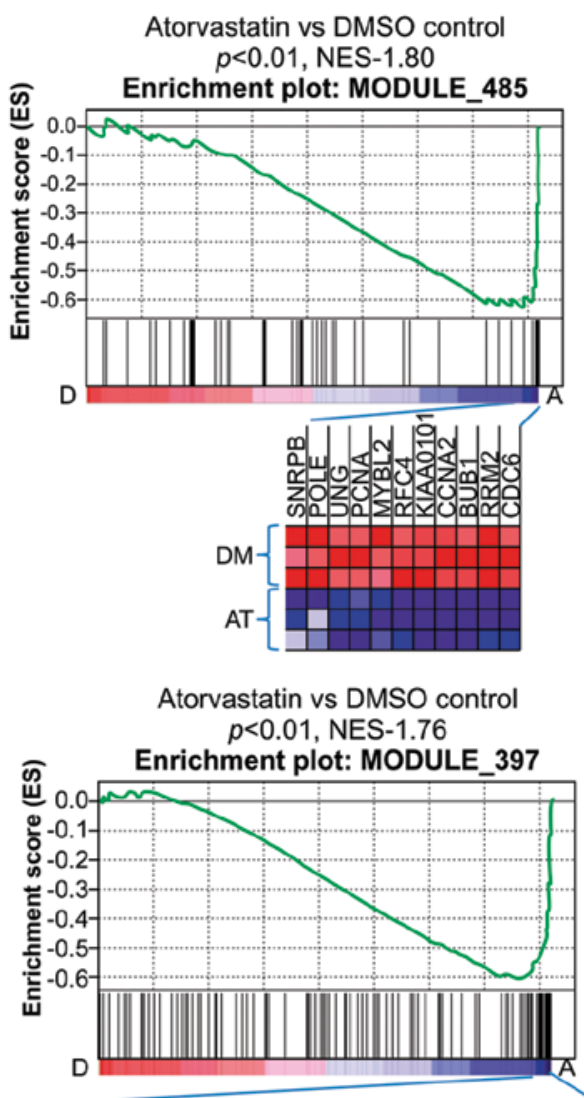

orvastatin vs DMSO control

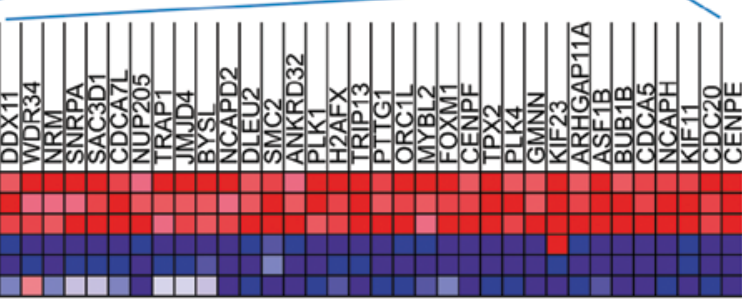

C
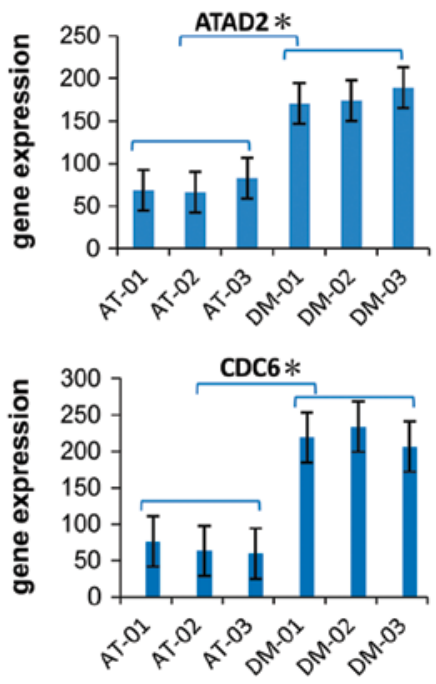

D
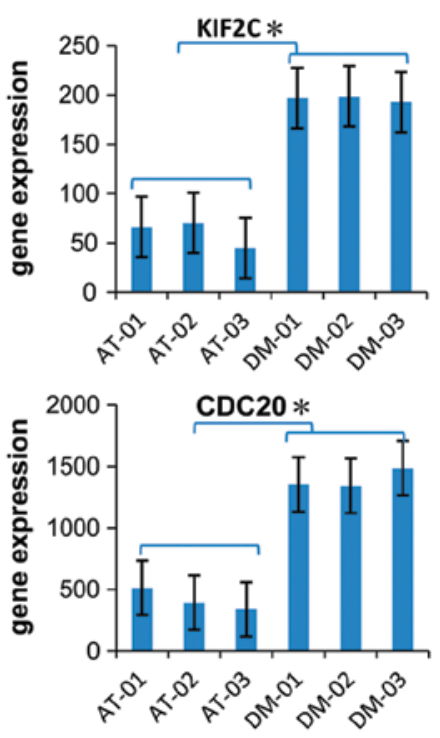

Figure 2. Downregulation of cancer modules in EA.hy926 cells. (A and B) Gene set enrichment analysis histograms of the gene set consisting of cancer modules 372, 485, 438 and 397. The enrichment score (ES; y-axis) reflects the degree to which a gene set was depressed with A, atorvastatin treatment or control treatment with D, dimethyl sulfoxide. Each solid bar represents 1 gene within a gene set. The heat-map image illustrates the gene expression levels of the leading edge subset. The normalized enrichment score (NES) and the nominal P-value are indicated. (C and D) Expression of representative genes (ATAD2, CDC6, KIF2C and CDC20) in modules 372, 485, 438 and 397. The significance was evaluated by the Student's t-test, "P<0.01. Data are the means \pm SD values of the 3 arrays. AT, atorvastatin; DM, dimethyl sulfoxide.

degree of pair-wise correlation in terms of the $\log 2$ gene expression. By contrast, the expression profiles of the 3 replicates were found to differ dramatically from those of the DMSO control group. Specifically, the results indicated that 295 genes were upregulated and 354 genes downregulated by $>2$-fold in the atorvastatin group compared with the DMSO group (Fig. 1).

Downregulation of cancer modules 397, 372, 485 and 438. According to the GSEA results, the gene sets in the MSigDB C4-designated modules 397, 372, 485 and 438, all of which are highly enriched in cancer cells compared with normal cells, were significantly downregulated in the atorvastatin group compared with the DMSO group (Fig. 2A and B). According to the Stanford University module map, liver cancer cell lines are enriched in the induced arrays of modules 397, 372, 485 and 438; B lymphoma cell lines in the induced genes of modules 438 and 485; hematological cancer, adenocarcinoma and breast cancer cell lines in the induced arrays of module 397; small cell lung cancer cell lines in the upregulated genes in modules 397 and 372; and P53-positive hepatocellular carcinoma cell lines in module 485 (16). Based on this map and the possibility that atorvastatin may repress the overexpression of genes in modules 397, 372, 485 and 438 compared with the DMSO control, it was concluded that atorvastatin had exerted an antitumor effect through these genes in the cancer modules.

The leading genes in the gene sets in the atorvastatin and DMSO groups were compared, including ATAD2, which is required for histone hyperacetylation and may be involved in the estrogen-induced cell proliferation and cell cycle progression of breast cancer cells; the cell division cycle (CDC) homolog 6 gene, which is involved in the initiation of DNA replication; the kinesin family member $2 \mathrm{C}$ gene (KIF2C), which promotes ATP-dependent removal of tubulin dimers from microtubules and regulates the microtubule turnover during the kinetochore and chromosome segregation that occurs during mitosis; and $\mathrm{CDC} 20$, which is required for 
A
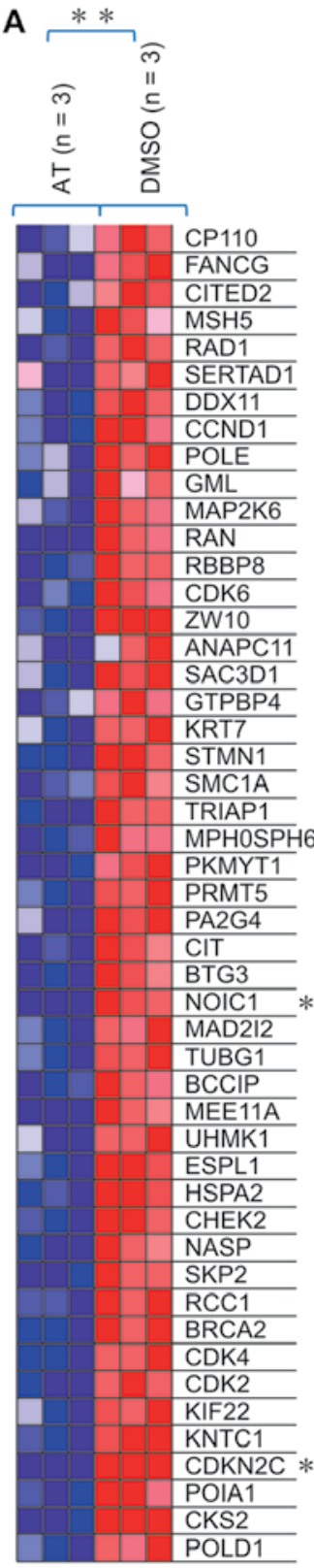
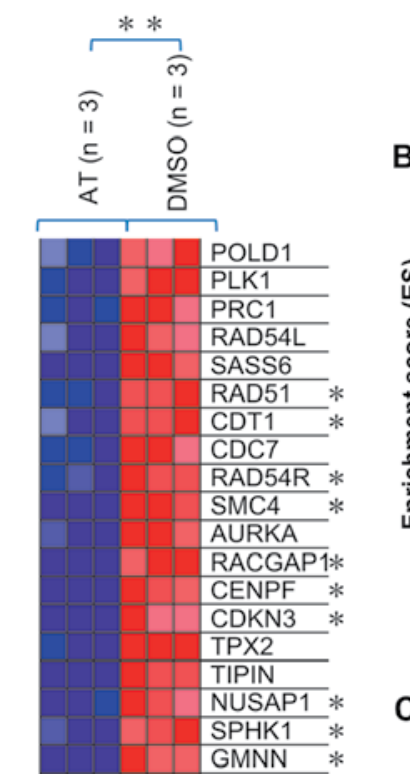

B
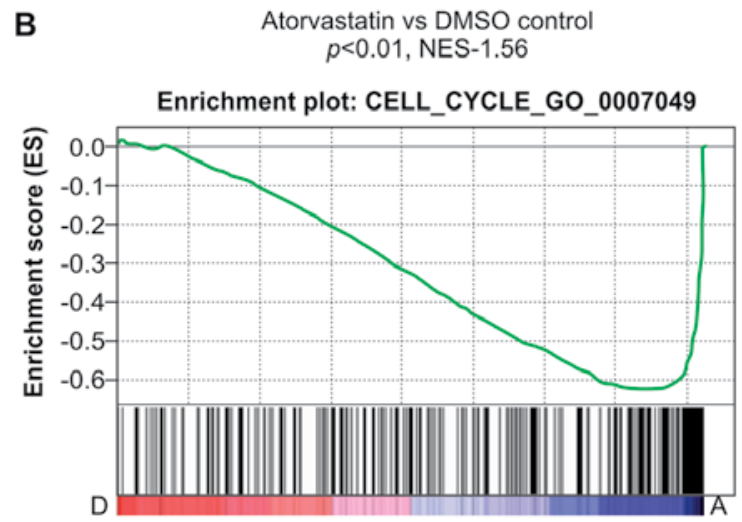

C

Atorvastatin vs DMSO control $p<0.01$, NES-1.64

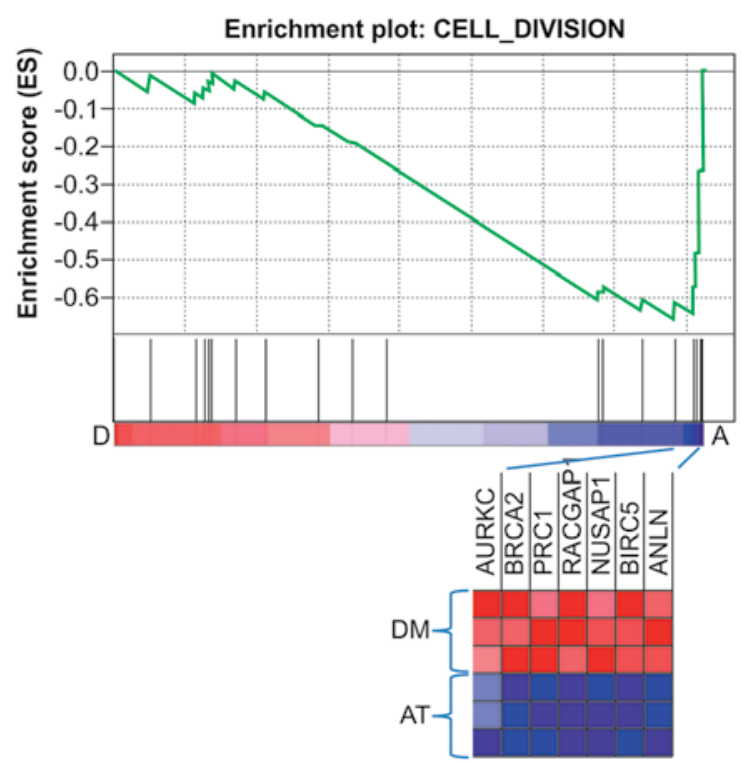

Figure 3. Downregulation of genes in the Gene Ontology cell cycle in EA.hy926 cells. (A and B) Gene set enrichment analysis histograms of the gene set associated with the cell cycle. The normalized enrichment score (NES) and nominal P-value are indicated. Genes that showed a significant difference $(\mathrm{P}<0.01)$ in expression levels between $\mathrm{A}$, the atorvastatin treatment and $\mathrm{D}$, dimethyl sulfoxide control groups and genes attaining statistical significance ( $\mathrm{P}<0.05$, fold change $>2.0$ ) are indicated. (C) Gene set enrichment analysis histogram of the gene set associated with the cell division.

nuclear movement prior to anaphase and chromosome separation, both of which are microtubule-dependent processes (Fig. 2C and D). According to the GO annotation of the cancer module gene sets, these genes are involved in DNA replication and microtubule genesis. Therefore, there is a possibility that atorvastatin regulates genes belonging to these cancer modules, inhibits DNA replication, and removes tubulin dimers from microtubules.

A consistent finding in the present study was that multiple gene sets related to the cell cycle regulation in C5 GO biological processes were also downregulated in the atorvastatin group compared with the DMSO group. Several cell cycle regulators, including $\mathrm{Cdk} 2, \mathrm{Cdk} 4, \mathrm{CDC} 25 \mathrm{~A}, \mathrm{CDC} 25 \mathrm{C}$, cyclin $(\mathrm{CCN})$ A2, CCNE2, CDC6, CDC7, CDCA5 and KIF11, essential in the control and promotion of the cell cycle at the G1/S (start) and the G2/M (mitosis) transitions, were also found to be downregulated in the atorvastatin group (Figs. 3A and B and 4).

Gene annotation enrichment analysis, BioCarta and KEGG pathway mapping. Analysis of the 354 genes downregulated by $>2$-fold in the atorvastatin group compared with the DMSO group using the DAVID functional annotation tool, revealed that the genes in annotation cluster 1 with the highest enrichment were involved in cell cycle regulation, cell division, microtubule cytoskeleton formation and mitosis (Fig. 5A). The BioCarta and KEGG pathways that were found to be significantly impacted by atorvastatin treatment are involved in the signaling of cancer and the regulation of the cell cycle 
A
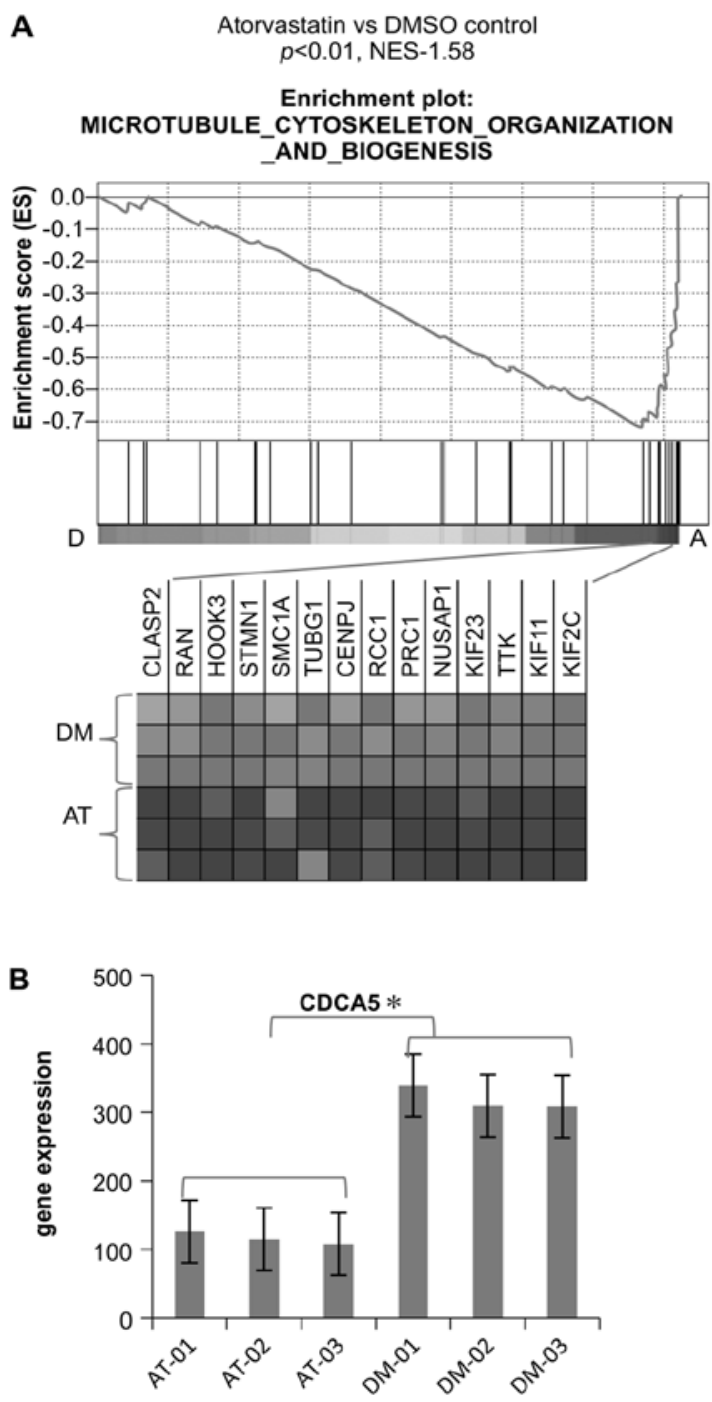

Atorvastatin vs DMSO control

$p<0.01$, NES- 1.58

Enrichment plot:

G1_S_TRANSITION_OF_MITOTIC_CELL_CYCLE
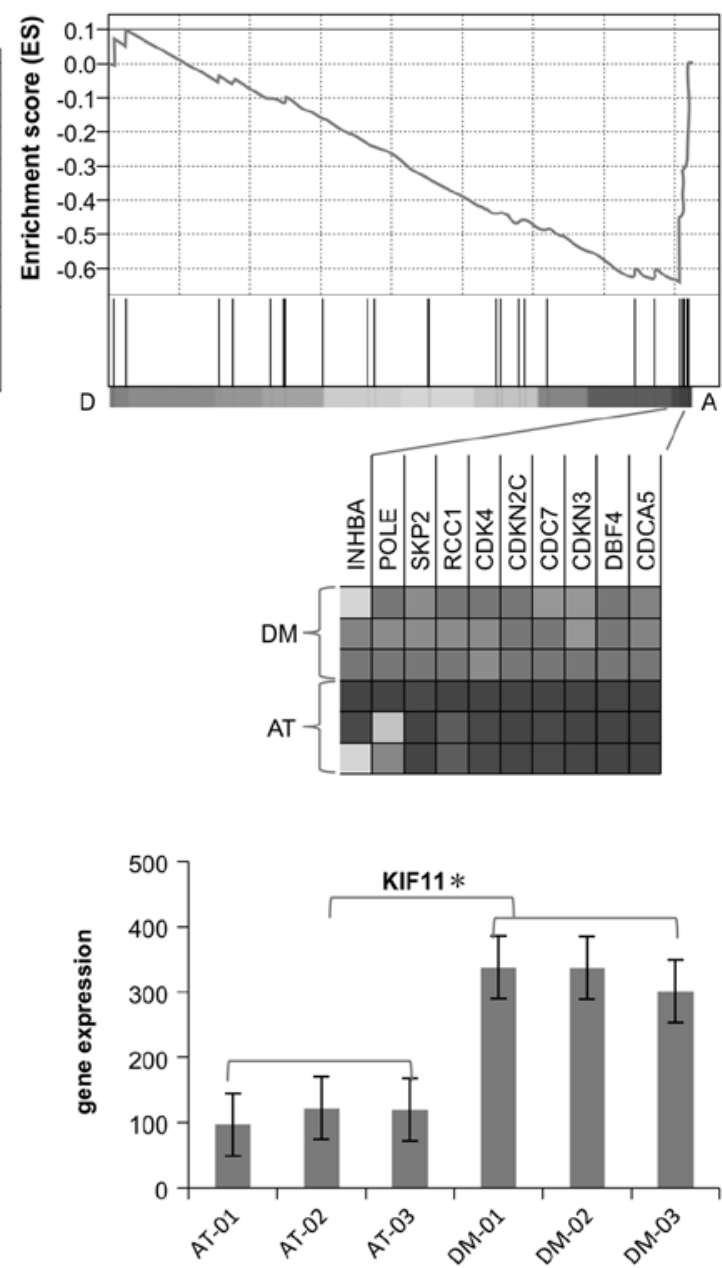

Figure 4. Downregulation of G1 to S transition and microtubule cytoskeleton organization and biogenesis regulators. (A) Gene set enrichment analysis histograms of the gene set associated with microtubule cytoskeleton organization and biogenesis and the gene set associated with G1 to S transition of the mitotic cell cycle. The normalized enrichment score (NES) and nominal P-value are indicated; A, refers to the atorvastatin treatment group and D, to the dimethyl sulfoxide control group. (B) Expression levels of the cell cycle regulators CDCA5 and KIF11. Significance was evaluated by the Student's t-test at P $<0.01$. Data are expressed as the means \pm SD values of the 3 arrays.

and cell metabolism (Fig. 5A). A comparison of the DAVID and GSEA results indicated that the cell cycle regulation and cyclin pathways were the most commonly affected pathways by atorvastatin treatment, suggesting that the mechanism underlying the anticancer pharmacological effect of atorvastatin is the negative regulation of the cell cycle. In addition, the gene-disease association indicated an association with cancer, particularly breast cancer (Fig. 5B).

Analysis of the 295 genes upregulated by $>2$-fold in the atorvastatin group compared with the DMSO group using the DAVID functional annotation tool at a medium level of gene functional classification stringency, revealed that the genes with the highest enrichment were involved in the regulation of transcription factors, specifically the Kruppel-like factors (KLFs) 2, 3, 4, 6, 7, 9 and 13. This finding of significant upregulation of KLFs in the atorvastatin group compared with the DMSO group is in accordance with recent in vitro and in vivo research demonstrating that KLFs suppress tumor growth in certain types of cancer $(17,18)$.
cMap analysis of related compounds. To identify related therapeutic compounds that may exert an effect on the gene expression similar to that of atorvastatin, gene expression analysis of 395 genes was performed, 155 of which were upregulated $>2$-fold and 240 of which were downregulated $>2$-fold in the atorvastatin group compared with the DMSO group. With the aim of identifying similar patterns in gene expression changes that may explain functional connections among drugs, genes, and diseases (19), 17 compounds whose profiles were highly similar to those of atorvastatin $(\mathrm{P}<0.001)$ were identified (Fig. 6A). Interestingly, the profile of the histone deacetylase (HDAC) inhibitor, MS-275, was mostly similar with the profiles of 2 other HDAC inhibitors (trichostatin A and vorinostat), also highly similar (Fig. 6B). In multiple instances, the profiles of Wnt/mTOR/PKC- $\delta$-ERK1/2 inhibitors, resveratrol and protein synthesis inhibitors were also highly similar to those of atorvastatin (Fig. 6A). Moreover, the results of cMap analysis performed to examine the profiles of simvastatin and lovastatin indicated that they positively 
A

\begin{tabular}{|c|c|c|c|c|c|c|}
\hline Annotation cluster 1 & Enrichment score: 45.39 & G & & Count & P_Value & Benjamini \\
\hline SP_PIR_KEYWORDS & cell cycle & RT & & 81 & $2.7 \mathrm{E}-64$ & $7.1 \mathrm{E}-62$ \\
\hline GOTERM_BP_FAT & cell cycle & RT & & 99 & $2.3 \mathrm{E}-60$ & $2.8 \mathrm{E}-57$ \\
\hline GOTERM_BP_FAT & M phase & RT & & 73 & $2.9 \mathrm{E}-90$ & $1.8 \mathrm{E}-57$ \\
\hline GOTERM_BP_FAT & cell cycle phase & RT & & 78 & $6.9 \mathrm{E}-59$ & $2.8 \mathrm{E}-56$ \\
\hline GOTERM_BP_FAT & nuclear division & RT & & 62 & $2.7 \mathrm{E}-57$ & $8.3 \mathrm{E}-55$ \\
\hline GOTERM_BP_FAT & mitosis & RT & & 62 & $2.7 \mathrm{E}-57$ & $8.3 \mathrm{E}-55$ \\
\hline GOTERM_BP_FAT & M phase of mitotic cell cycle & RT & & 62 & $9.4 \mathrm{E}-57$ & $2.3 E-54$ \\
\hline GOTERM_BP_FAT & organelle fission & RT & & 62 & $4.3 \mathrm{E}-56$ & $8.8 \mathrm{E}-54$ \\
\hline SP_PIR_KEYWORDS & mitosis & RT & & 55 & $5.7 \mathrm{E}-56$ & $7.5 E-54$ \\
\hline SP_PIR_KEYWORDS & cell division & RT & & 61 & $6.3 \mathrm{E}-55$ & $5.5 \mathrm{E}-53$ \\
\hline GOTERM_BP_FAT & cell cycle process & RT & & 83 & $4.9 \mathrm{E}-54$ & 8.6E-52 \\
\hline GOTERM_BP_FAT & mitotic cell cycle & RT & & 70 & $2.1 \mathrm{E}-52$ & $3.2 \mathrm{E}-50$ \\
\hline GOTERM_BP_FAT & cell division & RT & & 58 & $7.0 \mathrm{E}-44$ & $9.7 E-42$ \\
\hline GOTERM_CC_FAT & spindle & RT & & 38 & $2.2 \mathrm{E}-33$ & $1.5 \mathrm{E}-31$ \\
\hline GOTERM_CC_FAT & microtubule cytoskeleton & RT & & 49 & $6.0 \mathrm{E}-22$ & $1.6 \mathrm{E}-20$ \\
\hline GOTERM_CC_FAT & cytoskeletal part & RT & & 53 & $6.1 \mathrm{E}-16$ & 9.4E-15 \\
\hline SP_PIR_KEYWORDS & cytoskeleton & RT & & 40 & $2.2 \mathrm{E}-14$ & $5.7 \mathrm{E}-13$ \\
\hline GOTERM_CC_FAT & cytoskeleton & RT & & 58 & $6.3 \mathrm{E}-12$ & $1.0 \mathrm{E}-10$ \\
\hline
\end{tabular}

\section{B}

\begin{tabular}{|c|c|c|c|c|c|c|c|}
\hline Category & Term & RT & Genes & Count & $\%$ & P_Value & Benjamini \\
\hline KEGG_PATHWAY & Cell cycle & RT & $\square$ & 26 & 9.7 & $1.1 \mathrm{E}-20$ & $6.7 \mathrm{E}-19$ \\
\hline KEGG_PATHWAY & DNA replication & RT & $\square$ & 16 & 6.0 & $5.3 \mathrm{E}-18$ & $1.6 \mathrm{E}-16$ \\
\hline KEGG_PATHWAY & Oocyte meiosis & RT & $\mathbf{a}$ & 14 & 5.2 & $4.0 \mathrm{E}-8$ & $8.0 \mathrm{E}-7$ \\
\hline KEGG_PATHWAY & Progesterone-mediated oocyte maturation & RT & 口 & 9 & 3.4 & $1.1 \mathrm{E}-4$ & $1.7 \mathrm{E}-3$ \\
\hline KEGG_PATHWAY & Pyrimidine metabolism & RT & - & 9 & 3.4 & $2.2 \mathrm{E}-4$ & $2.7 \mathrm{E}-3$ \\
\hline KEGG_PATHWAY & Mismatch repair & RT & - & 5 & 1.9 & $6.0 \mathrm{E}-4$ & $6.1 \mathrm{E}-3$ \\
\hline KEGG_PATHWAY & p53 signaling pathway & RT & 口 & 6 & 2.2 & $6.4 \mathrm{E}--3$ & $5.5 \mathrm{E}-2$ \\
\hline KEGG_PATHWAY & Nucleotide excision repair & RT & घ & 5 & 1.9 & $7.0 \mathrm{E}-3$ & $5.2 \mathrm{E}-2$ \\
\hline KEGG_PATHWAY & Purine metabolism & RT & 口 & 7 & 2.6 & $5.1 \mathrm{E}-2$ & $3.0 \mathrm{E}-1$ \\
\hline KEGG_PATHWAY & RNA degradation & RT & 口 & 4 & 1.5 & $7.7 \mathrm{E}-2$ & $3.9 \mathrm{E}-1$ \\
\hline
\end{tabular}

\begin{tabular}{|l|l|c|c|c|c|c|c|}
\hline Category & Term & RT & Genes & Count & $\%$ & P_Value & Benjamini \\
\hline BIOCARTA & Role of Ran in mitotic spindle regulation & RT & $\mathbf{I}$ & 5 & 1.9 & $8.9 \mathrm{E}-5$ & $3.7 \mathrm{E}-3$ \\
\hline BIOCARTA & CDK Regulation of DNA Replication & RT & $\mathbf{I}$ & 3 & 1.1 & $1.2 \mathrm{E}-2$ & $2.3 \mathrm{E}-1$ \\
\hline BIOCARTA & Cyclins and Cell Cycle Regulation & RT & $\mathbf{U}$ & 4 & 1.5 & $1.7 \mathrm{E}-2$ & $2.1 \mathrm{E}-1$ \\
\hline BIOCARTA & How progesterone Initiates the Oocyte Maturation & RT & $\mathbf{U}$ & 3 & 1.1 & $7.1 \mathrm{E}-2$ & $5.4 \mathrm{E}-1$ \\
\hline
\end{tabular}

\begin{tabular}{|l|l|l|c|c|l|c|c|}
\hline Category & Term & RT & Genes & Count & $\%$ & P_Value & Benjamini \\
\hline GENETIC_ASSOCIATION_DB_DISEASE_CLASS & CANCER & RT & & 25 & 9.3 & $3.1 \mathrm{E}-5$ & $4.6 \mathrm{E}-4$ \\
\hline GENETIC_ASSOCIATION_DB_DISEASE & breast cancer & RT & & 14 & 5.2 & $9.8 \mathrm{E}-5$ & $9.3 \mathrm{E}-3$ \\
\hline
\end{tabular}

Figure 5. Gene clusters, BioCarta and KEGG pathways most highly enriched with differentially expressed genes. Network screenshot of results of the Database for Annotation, Visualization and Integrated Discovery analysis revealing the gene clusters, and that BioCarta and KEGG pathways are most highly enriched with differentially expressed genes.

correlated with the profiles of trichostatin A and vorinostat, a result that accords with that of a prior study that examined the chemical structure of statins (20).

Validation of microarray gene expression findings. The differential expression of 4 genes in the cell cycle pathway and 7 transcription factor genes were validated by quantitative RT-PCR on samples obtained from EA.hy926 cells that had been treated with atorvastatin and DMSO in a separate experi- ment. The selected genes were CCNB2, CCNB1, CCNA2 and CCNE2 and the selected transcription factors were KLFs 2, 3, 4, 6, 7, 9 and 13 . Whereas the PCR results and the microarray analysis results were concordant regarding the magnitude of the changes in the expression of the genes examined (Fig. 7), the PCR results indicated that the magnitude of the change in the expression of various transcription factors (KLFs 2, 3, 4, 7 and 13) examined was greater compared to the microarray analysis results. 


A
\begin{tabular}{|l|l|l|c|c|c|c|c|}
\hline Rank & Compound & Target/function & $\begin{array}{c}\text { Mean } \\
\text { connectivity score }\end{array}$ & $\begin{array}{c}\text { No of } \\
\text { Instances }\end{array}$ & Enrichment & p & Specificity \\
\hline 1 & MS-275 & HDAC inhibitor & 0.945 & 2 & 0.999 & 0 & 0.0087 \\
\hline 2 & resveratrol & Antioxidants & 0.523 & 9 & 0.758 & 0 & 0.0343 \\
\hline 3 & thioridazine & Phenothiazine & 0.599 & 20 & 0.72 & 0 & 0.0274 \\
\hline 4 & prochlorperazine & Phenothiazine & 0.581 & 16 & 0.708 & 0 & 0.0097 \\
\hline 5 & trifluoperazine & Phenothiazine & 0.521 & 16 & 0.646 & 0 & 0.0289 \\
\hline 6 & trichostatin A & HDAC inhibitor & 0.463 & 182 & 0.582 & 0 & 0.1564 \\
\hline 7 & sirolimus & mTOR inhibitor & 0.303 & 44 & 0.458 & 0 & 0.0843 \\
\hline 8 & fulvestrant & Estrogen Receptor Antagonist & 0.236 & 40 & 0.406 & 0 & 0 \\
\hline 9 & beta-escin & Antiproliferative & 0.521 & 6 & 0.848 & 0.00002 & 0 \\
\hline 10 & fluphenazine & Phenothiazine & 0.437 & 18 & 0.535 & 0.00004 & 0.0311 \\
\hline 11 & astemizole & H 1-blocking antihistamine & 0.691 & 5 & 0.891 & 0.00006 & 0.0332 \\
\hline 12 & dipyridamole & Thromboxane synthase inhibitor & 0.501 & 6 & 0.824 & 0.00006 & 0 \\
\hline 13 & prenylamine & Calcium channel blocker & 0.651 & 4 & 0.905 & 0.00008 & 0.0197 \\
\hline 14 & pyrvinium & Wnt inhibitor & 0.653 & 6 & 0.823 & 0.00008 & 0.0279 \\
\hline 15 & vorinostat & HDAC inhibitor & 0.401 & 12 & 0.593 & 0.00012 & 0.3266 \\
\hline 16 & puromycin & Protein synthesis inhibitor & 0.64 & 4 & 0.885 & 0.00018 & 0.0843 \\
\hline 17 & rottlerin & PKC-delta-ERK1/2 inhibitor & 0.615 & 3 & 0.94 & 0.0003 & 0.0259 \\
\hline
\end{tabular}

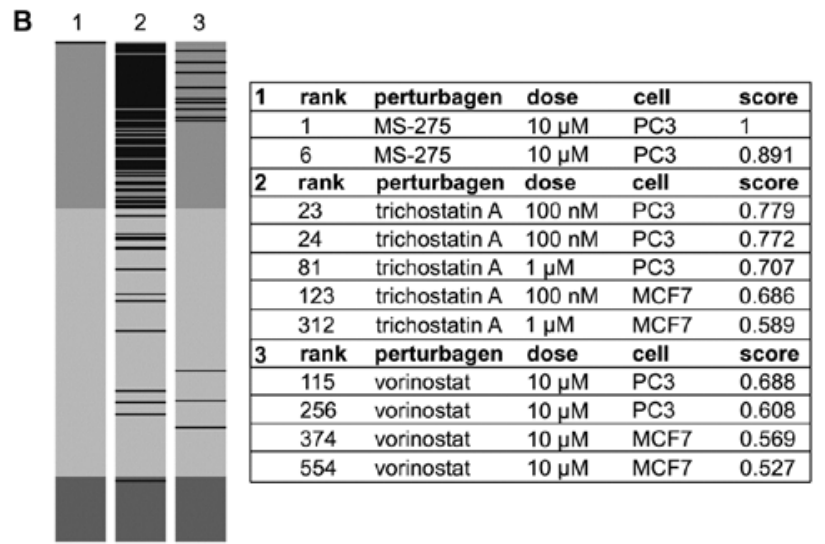

Figure 6. Results of gene expression correlation analysis. (A) The gene expression signatures of compounds most positively correlated with the gene expression signature of atorvastatin. Enrichment of both the upregulated and downregulated genes from a given signature was estimated with a metric based on Kolmogorov-Smirnov statistics and combined to produce a connectivity score. (B) Connectivity mapping of HDAC inhibitors. The bar view is constructed of 6,100 horizontal lines each representing an individual treatment instance ordered by its corresponding connectivity scores with signatures produced from the compounds MS-275, trichostatin A, and vorinostat. The upper gray part indicates a positive score; the middle one, a null score; and the lower one, a negative score (left panel). Part of the rank, name of the perturbagen, dose, cell line and connectivity score for each HDAC inhibitor are also shown (right panel).
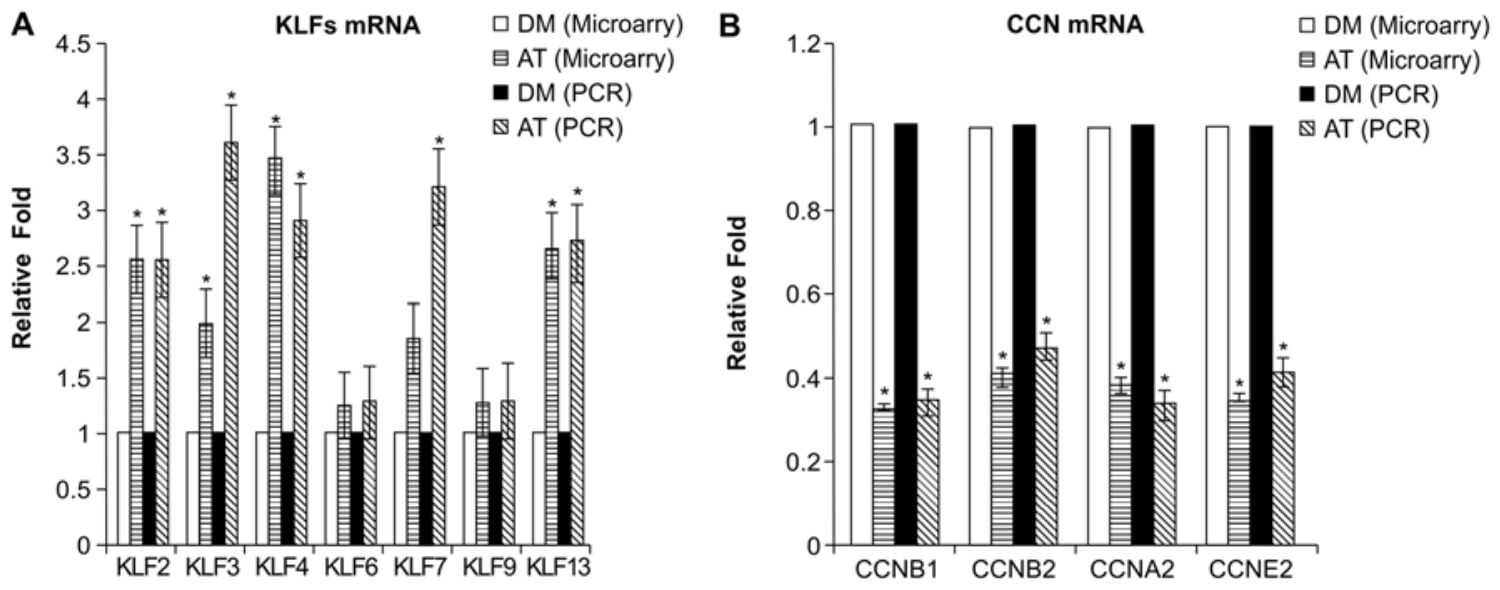

Figure 7. Analysis of changes in gene expression by microarray analysis and real-time PCR. (A and B) Incubation of EA.hy926 endothelial cells with $10 \mu \mathrm{M}$ of atorvastatin for $24 \mathrm{~h}$ increased Kruppel-like factor (KLF) upregulation and CCN mRNA expression. Data are normalized to GAPDH and the value for the $\mathrm{KLF}$ and cylcin $(\mathrm{CCN})$ expression in the control (dimethyl sulfoxide) group was set to 1 . Data are expressed as the means \pm SD of the 3 arrays. AT, atorvastatin group; DM, dimethyl sulfoxide group; ${ }^{*} \mathrm{P}<0.05$ AT vs. DM group.

\section{Discussion}

In recent years, statins have been widely prescribed for middleaged and elderly patients as part of a life-long therapeutic regimen, calling for greater research into their safety and toxicity. To date, most retrospective research has found no statistically significant increase in the risk of cancer or metastasis with statin use (21-23). Furthermore, laboratory analysis 
has indicated that hydrophobic statins, such as simvastatin and fluvastatin, but not hydrophilic pravastatin may inhibit the cancer cell progression via alteration of the equilibrium between pro- and anti-inflammatory cytokines (24) or the downregulation of SATB1 (25) in colon cancer cells and the inhibition of NFKB and Raf/MEK pathways in pancreatic cancer cells (26).

While the results of population studies remain controversial, the results of several clinical trials have suggested that long-term use of statins may reduce the risk of gastric cancer (27) and is associated with less advanced tumor stage or lower frequency of distant metastases in patients with colorectal cancer $(28,29)$ or prostate cancer (30). However, the results of other clinical trials and meta-analyses of these trials have indicated that statin use does not prevent cancer (31-33), while the results of other trials have suggested that statins have a dichotomous effect, being either cancer-inhibiting or promoting in certain environments $(6,34)$. Several factors may account for the conflicting clinical results, including the examination of different populations in different studies, the use of relatively short-term follow-up periods, and the use of a design in which the primary endpoint is not cancer development.

As statins are widely prescribed worldwide for increasingly longer periods, it is extremely important to comprehensively examine the possible applications of statins in cancer therapy. The results of recent in vitro studies, which are relatively consistent, have indicated that statins may exert an anticancer effect on cancer cells via multiple mechanisms. As endothelial recruitment and endothelial interactions in the tumor microenvironment are considered critical in tumorigenesis and cancer metastasis (35), examining the impact of statin therapy on endothelial cells may reveal the anticancer mechanism of statins. To satisfy this research requirement, the present study conducted DNA microarray analysis and drug-induced genome-wide transcriptional mapping, which is used to examine the action of drug targets and predict adverse effects (36), of cell samples taken from the endothelial cell line, EA.hy926.

The results of the microanalysis indicated that treatment with $10 \mu \mathrm{M}$ of atorvastatin induced the upregulation or downregulation of 649 genes by 2 -fold compared to treatment with DMSO, the control treatment. The results of gene function annotation and pathway analyses using GSEA and the DAVID functional annotation tool database were consistent, both indicating that atorvastatin represses the expression of genes enriched in cancer modules 397, 372, 485 and 438, which are involved in DNA replication and microtubule cytoskeleton formation. These genes, which include CDC6, CDC20, CDC7, CDC25A, CDC25C, CCNA2 and CCNE2, are essential in the control of the cell cycle at the G1/S (start) and the G2/M (mitosis) transitions. These results suggest that atorvastatin inhibits the endothelial cell cycle through multiple targets and, since endothelial cells are widely distributed in vivo, may play a role in tumor formation and metastasis in many cancers. The results of PCR analysis, which was performed to confirm the downregulation of genes involved in regulation of the G1/S and G2/M transitions, such as CCNA2, CCNE2, CCNB1 and $\mathrm{CCNB} 2$, are in agreement with the results of the microarray analysis. Moreover, the results of both real-time PCR and microarray analysis of the effect of atorvastatin on transcription factors, which confirmed the reliability of the GeneChip data, indicated that atorvastatin enhanced the expression of several KLFs, which can both activate and repress genes that participate in cell cycle regulation. Among the different KLFs, KLFs 2, 4, 6,9 and 10 have important tumor-suppressing functions $(37,38)$.

The cMap results indicated that the gene expression signature of atorvastatin positively correlated with that of HDAC, proteasome and Wnt inhibitors, and particularly with the gene expression signatures of the HDAC inhibitors, MS-275, trichostatin A and vorinostat, whose signatures were mostly similar to the signature of atorvastatin. These results, which indicate that atorvastatin may serve as an HDAC inhibitor in cancer therapy, are supported by those of a previous study, which also performed computational modeling, and found that the carboxylic acid moiety of statins directly interacted with the catalytic site of HDAC2 and, in a subsequent assay, that statins inhibited HDAC2 activity, indicating that they may exert an antitumor effect as HDAC inhibitors (39). Although only cancer cell lines are included in the cMap, the drug-induced gene expression profiles have been found to be concordant across cell lines, tissues and even organ systems, as signatures are often conserved across diverse cell types and settings $(13,36)$.

In conclusion, the results of this cell-based microarray and bioinformatic analysis of atorvastatin and related compounds using GSEA, the DAVID functional annotation tool, and the cMap database provide great insights into the molecular mechanism underlying the impact of atorvastatin therapy on endothelial cells and the connection between regulatory pathways at different phases of the cell cycle, all of which support the use of statins in cancer therapy. Specifically, statins exert an antitumor effect by promoting the upregulation of KLFs and the downregulation of cell cycle-related genes, such as CCNA2, CCNE2, CCNB1 and CCNB2, which inhibits both the G1/S and $\mathrm{G} 2 / \mathrm{M}$ transitions and forms a network of tumor suppressors. Detailed analysis of the differences in gene expression between the atorvastatin and DMSO control groups indicates that HDAC inhibitors may also exert a tumor-suppressive effect, either when used synergistically with or as substitutes for atorvastatin. These data may be further used to investigate the anticancer mechanism of statins in cancer cells and animals. Subsequent prospective clinical trials should be performed to confirm the beneficial effects of statins in cancer therapy.

\section{Acknowledgements}

This study was supported by the National Science and Technology Support Program (no. 2009BAI86B04).

\section{References}

1. Spampanato C, De Maria S, Sarnataro M, et al: Simvastatin inhibits cancer cell growth by inducing apoptosis correlated to activation of Bax and down-regulation of BCL-2 gene expression. Int J Oncol 40: 935-941, 2012.

2. Samson RH and Nair DG: Influence and critique of the JUPITER Trial (Statins v no statins for primary prevention of cardiovascular events in patients with normal lipids and elevated C-reactive protein). Semin Vasc Surg 24: 172-179, 2011.

3. Ridker PM, Danielson E, Fonseca FA, et al: Rosuvastatin to prevent vascular events in men and women with elevated C-reactive protein. N Engl J Med 359: 2195-2207, 2008.

4. Rossebo AB, Pedersen TR, Boman K, et al: Intensive lipid lowering with simvastatin and ezetimibe in aortic stenosis. N Engl J Med 359: 1343-1356, 2008. 
5. Marelli C, Gunnarsson C, Ross S, et al: Statins and risk of cancer: a retrospective cohort analysis of 45,857 matched pairs from an electronic medical records database of 11 million adult Americans. J Am Coll Cardiol 58: 530-537, 2011.

6. Chen ZY, Rex S and Tseng CC: Kruppel-like factor 4 is transactivated by butyrate in colon cancer cells. J Nutr 134: 792-798, 2004.

7. Simon MS, Rosenberg CA, Rodabough RJ, et al: Prospective analysis of association between use of statins or other lipidlowering agents and colorectal cancer risk. Ann Epidemiol 22: 17-27, 2012.

8. Yang YC, Huang WF, Chuan LM, et al: In vitro and in vivo study of cell growth inhibition of simvastatin on chronic myelogenous leukemia cells. Chemotherapy 54: 438-446, 2008.

9. Gauthaman K, Fong CY and Bongso A: Statins, stem cells, and cancer. J Cell Biochem 106: 975-983, 2009.

10. Upreti M, Koonce NA, Hennings L, Chambers TC and Griffin RJ: Pegylated IFN-alpha sensitizes melanoma cells to chemotherapy and causes premature senescence in endothelial cells by IRF-1 mediated signaling. Cell Death Dis 1: e67, 2010.

11. Subramanian A, Tamayo P, Mootha VK, et al: Gene set enrichment analysis: a knowledge-based approach for interpreting genome-wide expression profiles. Proc Natl Acad Sci USA 102: 15545-15550, 2005.

12. National Institute of Allergy and Infectious Diseases. DAVID Bioinformatics Resources 6.7. http://david.abcc.ncifcrf.gov. Accessed January 13, 2012

13. Lamb J, Crawford ED, Peck D, et al: The Connectivity Map: using gene-expression signatures to connect small molecules, genes, and disease. Science 313: 1929-1935, 2006.

14. Broadband Institute. Molecular Signatures Database http://www. broadinstitute.org/gsea/msigdb/indexjsp. Accessed January 13, 2012.

15. Broadband Institute. Map C. http://www.broadinstitute.org/ cmap. Accessed January 13, 2012.

16. Stanford University. Index of cancer modules. http://robotics. stanford.edu/ erans/cancer/modules. Accessed January 13, 2012.

17. Taniguchi H, Jacinto FV, Villanueva A, et al: Silencing of Kruppel-like factor 2 by the histone methyltransferase EZH2 in human cancer. Oncogene 31: 1988-1994, 2012.

18. Yori JL, Seachrist DD, Johnson E, et al: Kruppel-like factor 4 inhibits tumorigenic progression and metastasis in a mouse model of breast cancer. Neoplasia 13: 601-610, 2011

19. Zimmer M, Lamb J, Ebert BL, et al: The connectivity map links iron regulatory protein-1-mediated inhibition of hypoxia-inducible factor-2a translation to the anti-inflammatory 15-deoxy-delta12,14-prostaglandin J2. Cancer Res 70 3071- 3079, 2010.

20. Lin YC, Lin JH, Chou CW, Chang YF, Yeh SH and Chen CC: Statins increase p21 through inhibition of histone deacetylase activity and release of promoter-associated HDAC1/2. Cancer Res 68: 2375-2383, 2008

21. Pandya K, Donze D and Townes TM: Novel transactivation domain in erythroid Kruppel-like factor (EKLF). J Biol Chem 276: 8239-8243, 2001

22. Funnell AP, Maloney CA, Thompson LJ, et al: Erythroid Kruppel-like factor directly activates the basic Kruppel-like factor gene in erythroid cells. Mol Cell Biol 27: 2777-2790, 2007.

23. Huang B, Ahn YT, McPherson L, Clayberger C and Krensky AM: Interaction of PRP4 with Kruppel-like factor 13 regulates CCL5 transcription. J Immunol 178: 7081-7087, 2007.
24. O'Grady E, Mulcahy H, Adams C, Morrissey JP and O'Gara F: Manipulation of host Kruppel-like factor (KLF) function by exotoxins from diverse bacterial pathogens. Nat Rev Microbiol 5: 337-341, 2007

25. Fisch S, Gray S, Heymans S, et al: Kruppel-like factor 15 is a regulator of cardiomyocyte hypertrophy. Proc Natl Acad Sci USA 104: 7074-7079, 2007.

26. Pilon AM, Nilson DG, Zhou D, et al: Alterations in expression and chromatin configuration of the alpha hemoglobin-stabilizing protein gene in erythroid Kruppel-like factor-deficient mice. Mol Cell Biol 26: 4368-4377, 2006.

27. Wang SP, Zhou HJ, Chen XP, et al: Loss of expression of Kruppel-like factor 6 in primary hepatocellular carcinoma and hepatoma cell lines. J Exp Clin Cancer Res 26: 117-124, 2007.

28. Chanchevalap S, Nandan MO, McConnell BB, et al: Kruppel-like factor 5 is an important mediator for lipopolysaccharide-induced proinflammatory response in intestinal epithelial cells. Nucleic Acids Res 34: 1216-1223, 2006.

29. Mukai S, Hiyama T, Tanaka S, Yoshihara M, Arihiro K and Chayama K: Involvement of Kruppel-like factor 6 (KLF6) mutation in the development of nonpolypoid colorectal carcinoma. World J Gastroenterol 13: 3932-3938, 2007.

30. Velarde MC, Zeng Z, McQuown JR, Simmen FA and Simmen RC: Kruppel-like factor 9 is a negative regulator of ligand-dependent estrogen receptor alpha signaling in Ishikawa endometrial adenocarcinoma cells. Mol Endocrinol 21: 2988-3001, 2007.

31. Chen ZY, Wang X, Zhou Y, Offner G and Tseng CC: Destabilization of Kruppel-like factor 4 protein in response to serum stimulation involves the ubiquitin-proteasome pathway. Cancer Res 65: 10394-10400, 2005.

32. Zhang XL, Zhang D, Michel FJ, Blum JL, Simmen FA and Simmen RC: Selective interactions of Kruppel-like factor 9/ basic transcription element-binding protein with progesterone receptor isoforms $\mathrm{A}$ and $\mathrm{B}$ determine transcriptional activity of progesterone-responsive genes in endometrial epithelial cells. J Biol Chem 278: 21474-21482, 2003.

33. Shie JL, Chen ZY, O'Brien MJ, Pestell RG, Lee ME and Tseng CC: Role of gut-enriched Kruppel-like factor in colonic cell growth and differentiation. Am J Physiol Gastrointest Liver Physiol 279: G806-G814, 2000.

34. Ghaleb AM, McConnell BB, Nandan MO, Katz JP, Kaestner KH and Yang VW: Haploinsufficiency of Kruppel-like factor 4 promotes adenomatous polyposis coli dependent intestinal tumorigenesis. Cancer Res 67: 7147-7154, 2007.

35. Schober SL, Kuo CT, Schluns KS, Lefrancois L, Leiden JM and Jameson SC: Expression of the transcription factor lung Kruppel-like factor is regulated by cytokines and correlates with survival of memory $\mathrm{T}$ cells in vitro and in vivo. J Immunol 163: 3662-3667, 1999.

36. Iskar M, Campillos M, Kuhn M, Jensen LJ, van Noort V and Bork P: Drug-induced regulation of target expression. PLoS Comput Biol 6: e1000925, 2010.

37. Guan H, Xie L, Leithauser F, et al: KLF4 is a tumor suppressor in B-cell non-Hodgkin lymphoma and in classic Hodgkin lymphoma. Blood 116: 1469-1478, 2010.

38. Bureau C, Hanoun N, Torrisani J, Vinel JP, Buscail L and Cordelier P: Expression and Function of Kruppel Like-Factors (KLF) in Carcinogenesis. Curr Genomics 10: 353-360, 2009.

39. Chnari E, Nikitczuk JS, Uhrich KE and Moghe PV: Nanoscale anionic macromolecules can inhibit cellular uptake of differentially oxidized LDL. Biomacromolecules 7: 597-603, 2006. 